\title{
CYTOKINE SCORE IDENTIFIES NICU PATIENTS WITH GRAM-NEGATIVE BACTEREMIA
}

\author{
L.L. Raynor ${ }^{1}$, J.J. Saucerman ${ }^{1}$, M.O. Akinola ${ }^{2}$, D.E. Lake ${ }^{1}$, J.R. Moorman ${ }^{1}$, K.D. Fairchild ${ }^{1}$ \\ ${ }^{1}$ University of Virginia, Charlottesville, VA, ${ }^{2}$ Wake Forest University, Winston-Salem, NC, USA
}

Background: Gram-negative late-onset neonatal sepsis has high mortality, but initial antibiotic regimens may not cover these most virulent pathogens. While heart rate characteristics (HRC) monitoring can lead to early sepsis diagnosis, other non-infective conditions elevate the HRC index, or HeRO score. Since a recent randomized trial showed reduced mortality with HeRO monitoring, we expect its use to increase. Cytokine levels rise in response to systemic inflammation and sepsis, and patterns of expression might differ depending on the infective organism.

Aims: We tested the hypothesis that patterns of plasma cytokine levels might identify HeRO-monitored NICU patients with Gram-negative late-onset sepsis.

Methods: 226 remnant plasma samples were collected from NICU patients $>3$ days old undergoing blood culture for sepsis suspected for any reason, including the HeRO score alone. 32 cultures were positive, 8 with Gram-negative bacteria. Seven cytokines were measured.

Results: All seven cytokines were significantly correlated with outcome (bacteremia, clinical sepsis, or sepsis ruled out), and two cytokines were correlated with HeRO score (IL-1 receptor antagonist and IL-8, $\mathrm{r}=0.31$ and 0.21 , respectively, $\mathrm{p}<0.001)$.

We developed a cytokine score using optimized thresholds for G-CSF, IL-6, IL-8, and TNF-alpha. If all 4 were elevated, there was $100 \%$ sensitivity and $62 \%$ positive predictive accuracy for Gram-negative bacteremia.

Conclusion: Early detection of neonatal sepsis is enhanced by cytokine measurements, especially for Gramnegative organisms. Early identification of Gram-negative bacteremia in infants suspected of subclinical illness could allow more rapid institution of appropriate antibiotics and adjunct sepsis therapies. 